\title{
Comprendiendo la resistencia a antibióticos
}

\author{
Jesús Oteo-Iglesias
}

Laboratorio de Referencia e Investigación en Resistencia a Antibióticos e Infecciones Relacionadas con la Asistencia Sanitaria, Centro Nacional de Microbiología, Instituto de Salud Carlos III; Red Española de Investigación en Patologías Infecciosas (REIPI RD16/0016), Instituto de Salud Carlos III; https://orcid.org/0000-0003-3327-8263; jesus.oteo@isciii.es

Recibido: 16/11/2019; Aceptado: 25/11/2019; Publicado: 29/11/2019

Resumen: El descubrimiento de los antibióticos supuso uno de los principales avances cualitativos de la historia de la medicina. Ahora, la diseminación de bacterias con resistencia a múltiples antibióticos se ha convertido en una amenaza global a la salud pública y a la salud individual de los pacientes; principalmente fundamentada en la aparición de bacterias resistentes a la práctica totalidad de los antibióticos y en la facilidad con la que son capaces de diseminarse. La resistencia a antibióticos es un fenómeno complejo, multifactorial y rápidamente evolutivo en el cual no está solamente implicado el ser humano, sino también la cadena alimentaria, el medio ambiente y los animales, tanto domésticos o de granja como salvajes. La OMS ha elaborado un listado de las principales bacterias resistentes a antibióticos que requieren investigación y desarrollo de nuevas estrategias de tratamiento. Las prioritarias son Pseudomonas. aeruginosa, Acinetobacter baumannii y las enterobacterias resistentes a antibióticos carbapenémicos. La investigación en nuevas alternativas terapéuticas, el desarrollo de métodos de diagnóstico rápidos que permitan iniciar un tratamiento precoz con antibiótico solo en las infecciones bacterianas que lo necesiten, la implementación de estrategias de vacunación globales, la realización de campañas educativas e informativas, la mejora en el control de la infección, el apoyo al proceso de modificación de los sistemas de cría y producción animal hacia un menor consumo de antibióticos en animales, entre otras, son estrategias necesarias que deben abordarse ya de forma coordinada a nivel mundial.

Palabras Clave: Resistencia a antibióticos, Biología bacteriana, “Única salud”, PRAN, PROA.

\begin{abstract}
The discovery of antibiotics was one of the main qualitative advances in the history of medicine. Now, the spread of bacteria with resistance to multiple antibiotics has become a global threat to public health and to the patient's health; mainly based on the appearance of bacteria resistant to almost all antibiotics and the ease with which they are able to spread. Antibiotic resistance is a complex, multifactorial and rapidly evolving phenomenon in which not only the human being is involved, but also the food chain, the environment and animals, both domestic or farm and wild. WHO has developed a list of the main antibiotic resistant bacteria that require research and development of new treatment strategies. The priorities are isolates resistant to carbapenem antibiotics of Pseudomonas aeruginosa, Acinetobacter baumannii and Enterobacteriaceae species. Some necessary strategies, among others, that must be tackled in a coordinated manner worldwide to fight against the antibiotic resistance are: 1) to research into new therapeutic alternatives, 2) to develop rapid diagnostic methods that allow early antibiotic treatment to be initiated only in bacterial infections, 3) to implement global vaccination strategies, 4) to carry out educational and information campaigns, 5) to improve the infection control programs, 6) to support for the process of modifying animal husbandry and production systems towards lower consumption of antibiotics in animals.
\end{abstract}

Key words: Antibiotic resistance, Bacterial biology, “One health", PRAN, PROA. 


\section{El impacto de la resistencia a los antibióticos}

La incorporación de los antibióticos a la práctica clínica supuso uno de los principales avances cualitativos de la historia de la medicina. El pronóstico de las infecciones bacterianas mejoró drásticamente, y muchos de los procedimientos sanitarios se modificaron de forma radical. Antes de que Alexander Fleming descubriera la penicilina, las enfermedades infecciosas eran la principal causa de muerte en el mundo, alrededor del $75-85 \%$ de los pacientes que sufrían una meningitis bacteriana moría, y la neumonía producida por bacterias tenía cifras de mortalidad cercanas al 40\% [1]. Pero el beneficio de los antibióticos no fue sólo directo, curando o evitando infecciones bacterianas que antes podían ser mortales, si no también indirecto; muchas de las más avanzadas y exitosas técnicas terapéuticas de la medicina actual, como las grandes cirugías, los trasplantes o la quimioterapia, serían inviables sin una cobertura antibiótica adecuada [1].

Por tanto, la creciente aparición y diseminación de bacterias con resistencia a múltiples familias de antibióticos se ha convertido en una amenaza global a la salud pública y a la salud individual de los pacientes [2]. Las bacterias pueden sobrevivir y multiplicarse en presencia de antibióticos generando infecciones más duraderas, con una mayor morbi-mortalidad y con un aumento significativo del gasto sanitario [3]. Un informe encargado por el gobierno británico hace unos años, Antimicrobial Resistance:Tackling a crisis for the health and wealth of nations [3], estimó la mortalidad actual anual debida a las bacterias resistentes en alrededor de 700.000 muertes al año, inferior al 1,2 y 8,2 millones de muertes que producen los accidentes de tráfico y el cáncer, respectivamente. Sin embargo, si la evolución de los patógenos con resistencia a múltiples antibióticos continúa al alza como en los últimos años, la mortalidad anual asociada a la resistencia podría alcanzar los 10 millones de muertes en 2050 [3].

En la actualidad, la lucha contra la resistencia a antibióticos está considerada una prioridad sanitaria por las principales instituciones tanto nacionales como internacionales [2,4]. La aparición de bacterias que acumulan resistencia a la práctica totalidad de los antibióticos que pueden ser utilizados frente a ellas y la facilidad con la que son capaces de diseminarse son sus principales amenazas.

\section{Biología bacteriana y resistencia}

Las bacterias poseen una enorme y rápida capacidad de adaptación al medio ambiente en el que viven. Una de sus grandes ventajas radica en la velocidad con la que se reproducen, una bacteria puede duplicar su población en tan solo media hora, y dar lugar a casi 50 generaciones de bacterias en un día. En esas condiciones, la selección natural facilita la adaptación de una población bacteriana a un medio hostil, como es un medio con antibióticos, en muy poco tiempo. Los antibióticos actúan como agentes selectores de aquellas mutaciones sufridas al azar que dotan a las bacterias de la ventaja selectiva de sobrevivir en presencia de antibióticos.

Los antibióticos solo se han utilizado en la práctica médica durante los últimos 70-80 años. Sin embargo, mediante estudios de metagenómica se han detectado genes muy similares a los actuales genes de resistencia a antibióticos $\beta$-lactámicos, aminoglucósidos y fluoroquinolonas, entre otros, en la momia de una mujer andina fallecida hace unos 3000 años [5]. Esta misma metodología ha demostrado la existencia de genes de resistencia en muestras de suelo que ha permanecido congelado durante los últimos 30.000 años, junto a genes de mamut y de otras especies del Pleistoceno [6].

Muchos de los antibióticos utilizados en clínica derivan de bacterias, sobre todo de bacterias ambientales entre las que predominan las denominadas Streptomyces. Para poder competir con éxito en ecosistemas con una alta densidad bacteriana y una lucha constante por los recursos, algunas bacterias desarrollaron la capacidad de producir sustancias que mataban a sus competidoras. Para que esta ventaja competitiva de producir antibióticos fuera eficaz, tuvo que ir necesariamente acompañada de la capacidad de sobrevivir en su presencia. Es decir, la aparición de los primeros genes de resistencia probablemente fuera sincrónica a la de los antibióticos, como adaptación a un medio con moléculas antibacterianas elaboradas por bacterias vecinas o como protección de las que ellas mismas producían [7]. La intervención del ser humano con la generalización del uso de antibióticos, la creación de nuevos antibióticos, y la modificación de otros generando fármacos de 
amplio espectro, ha creado un nuevo escenario que ha acelerado y descontrolado procesos biológicos ya existentes.

Hay dos factores que agravan la amenaza de la resistencia a antibióticos a nivel mundial. Uno de ellos es la capacidad bacteriana de acumular diferentes mecanismos de resistencia. En la actualidad hay bacterias que tienen una dotación genética que les permite sobrevivir en presencia de todos los antibióticos disponibles, son las bacterias denominadas pan-resistentes frente a las cuales no tenemos un tratamiento antibiótico adecuado [8]. El otro es su gran capacidad de diseminación entre diferentes pacientes, instituciones y regiones geográficas. La existencia de clones multirresistentes de alto riesgo epidemiológico, como son el ST258 de Klebsiella pneumoniae [9] o el ST175 de Pseudomonas aeruginosa [10], de plásmidos epidémicos portadores de varios genes de resistencia [9] que se transfieren entre bacterias incluso de diferentes especies, y la existencia de personas sanas colonizadas por bacterias multirresistentes, cuya detección y control no siempre son fáciles, contribuyen a dicha dispersión [11].

\section{La resistencia a antibióticos como paradigma del concepto de una "Única Salud"}

La resistencia a antibióticos es un fenómeno complejo, multifactorial y rápidamente evolutivo en el cual no está solamente implicado el ser humano, sino también la cadena alimentaria, el medio ambiente y los animales, tanto domésticos o de granja como salvajes [12,13].

Hace unos años, el estudio de las aguas de un río de India [14], al que vertían las aguas residuales de fábricas de producción de antibióticos, detectó niveles muy altos de varias clases de genes de resistencia, así como de elementos genéticos móviles para su transferencia horizontal (integrones, transposones y plásmidos). La existencia de una planta depuradora previa a su vertido no evitaba que las concentraciones de antibióticos río abajo de esas fábricas fueran alarmantemente altas y muy superiores a las encontradas río arriba. Este hecho influía significativamente en las poblaciones de bacterias ambientales, diferentes y mucho más diversas río arriba que río abajo del vertido de las fábricas [14].

En un estudio realizado recientemente en 668 aves salvajes en España [15], se detectó un 8,7\% de aves (58 individuos pertenecientes a 28 especies diferentes) colonizadas por enterobacterias productoras de $\beta$-lactamasas de espectro extendido de las mismas familias que predominan en humanos (CTX-M-15, CTX-M-9, CTX-M-14 y SHV-12). Además, se identificaron 5 individuos colonizados por enterobacterias productoras de la carbapenemasa OXA-48 pertenecientes a algunos de los clones detectados con más frecuencias en clínica humana [15]. La diversidad de hábitos alimenticios y migratorios de las diferentes especies de aves que pueden portar bacterias multirresistentes podría contribuir a su diseminación global desde fuentes de origen humano.

La colistina se ha convertido en uno de los tratamientos de última línea para infecciones por bacterias gramnegativas con resistencia a múltiples antibióticos. La transmisión de los genes plasmídicos $\mathrm{mor}$ de resistencia a colistina desde animales de granja, su presumible origen, al ser humano supone una amenaza sanitaria de primer orden [16]. Se trata solo de algunos ejemplos que ponen de manifiesto la necesidad de luchar contra la resistencia a antibióticos implementando iniciativas integrales y coordinadas a diferentes niveles con una perspectiva de una única salud.

\section{Principales amenazas sanitarias en el campo de la Resistencia a antibióticos}

La Organización Mundial de la Salud ha elaborado recientemente un listado de las principales bacterias resistentes a antibióticos que requieren investigación y desarrollo de nuevas estrategias de tratamiento [17]. Las prioritarias son algunas bacterias gramnegativas frente a las cuales apenas tenemos opciones terapéuticas, entre ellas se encuentran P. aeruginosa, Acinetobacter baumannii y las enterobacterias resistentes a antibióticos carbapenémicos [17]. La resistencia a estos antibióticos, considerados unos de los tratamientos de última línea frente a las infecciones por bacilos gramnegativos, supone un muy buen indicador de la presencia de multirresistencia (resistencia a tres o más familias de antibióticos) o de resistencia extensa a los antibióticos (resistencia a todos los antibióticos excepto a uno o dos) [18]. La resistencia a antibióticos carbapenémicos en enterobacterias se debe fundamentalmente a la producción de unas enzimas que hidrolizan la práctica totalidad de 
los antibióticos $\beta$-lactámicos: las carbapenemasas [19]. Las enterobacterias productores de carbapenemasas (EPC) constituyen un formidable desafío para los sistemas de salud de todo el mundo, están en continua evolución y presentan una alta capacidad de diseminación de difícil control. Con frecuencia, la producción de carbapenemasas se asocia a resistencia a otros antibióticos limitando las alternativas terapéuticas. La enterobacteria K. pneumoniae productora de la carbapenemasa KPC está ampliamente en muchos lugares del mundo entre los que se encuentra la isla de Creta. En 2010, los aislados de K. pneumoniae productores de KPC en Creta presentaban resistencia a todos los antibióticos excepto a la colistina y la tigeciclina [20]. Como consecuencia, las infecciones producidas por esas bacterias se trataron con dichos antibióticos. La presión selectiva generada por ellos aumentó, y en 2014 hasta una cuarta parte de los aislados habían adquirido resistencia también a colistina y tigeciclina [20], es decir, se habían transformado en bacterias panresistentes [18] frente a las cuales no había antibiótico eficaz. No es más que es un ejemplo de la evolución que está experimentando este tipo de bacterias a nivel mundial.

\section{Luchando contra la Resistencia a Antibióticos}

Aunque la dispersión mundial de la resistencia a antibióticos tiene un origen multifactorial, la incorrecta utilización de los antibióticos y su uso excesivo, tanto en humanos como animales, es el principal factor que facilita y condiciona dicho proceso. Según los resultados publicados por la encuesta del Eurobarómetro en 2018 [21], una tercera parte de los europeos hemos tomado antibióticos en los últimos 12 meses; en España esta cifra se encuentra por encima de la media europea alcanzando el $42 \%$ [21].

Se puede conseguir un uso más responsable y prudente de los antibióticos actuando sobre su prescripción, sobre su venta y sobre la forma en la que se toman. Uno de los principales problemas en la utilización de antibióticos es su uso para infecciones de vías respiratorias altas, en su gran mayoría producidas por virus frente a los que los antibióticos no son eficaces. Su incorrecto uso en profilaxis, que requiere diferentes pautas de administración y periodos más cortos que los tratamientos, también es un factor a mejorar en cirugías e instrumentaciones.

En España, toda esta problemática llevó en 2014 a la creación del Plan Nacional frente a la Resistencia a los Antibióticos (PRAN). Se trata de un plan estratégico y de acción cuyo objetivo es reducir el riesgo de selección y diseminación de resistencia a los antibióticos, preservando de manera sostenible la eficacia de los antibióticos existentes [4]. El PRAN tiene entre sus objetivos la implantación de los Programas de Optimización de Uso de los Antibióticos (PROA) [4,22] tanto en el ámbito hospitalario como en el de atención primaria. Estos programas trabajan en la optimización de la prescripción de antibióticos para mejorar el pronóstico de los pacientes que los necesitan, minimizar los efectos adversos, controlar la aparición de resistencia y garantizar el uso de tratamientos coste-eficaces. En estrecha relación con los PROA, los programas para el control de la infección son también claves en la contención de la diseminación de las bacterias resistentes.

Solo podemos luchar eficazmente contra la multirresistencia mediante una actuación coordinada de todos los actores implicados como son, entre muchos otros, los consumidores, los profesionales sanitarios y las instituciones nacionales y supranacionales. La investigación en nuevos antibióticos y nuevas alternativas terapéuticas, el desarrollo de métodos de diagnóstico rápidos que permitan iniciar un tratamiento precoz con antibiótico solo en las infecciones bacterianas que lo necesiten, la implementación de estrategias de vacunación globales que disminuyan el consumo de antibióticos, la realización de campañas educativas e informativas sobre el uso adecuado de los antibióticos, la mejora en el control de la infección, el apoyo al proceso de modificación de los sistemas de cría y producción animal hacia un menor consumo de antibióticos en animales, entre otras, son estrategias necesarias que deben abordarse ya de forma coordinada y a nivel mundial (Tabla 1) [3].

La evolución de los diferentes factores implicados en la resistencia a antibióticos nos ha llevado a una encrucijada crítica. El ECDC estimó en casi 875.000 los años perdidos por enfermedad, discapacidad o muerte prematura debidos a infecciones producidas por bacterias resistentes a los antibióticos en Europa en 2015 [23]. La evolución de los acontecimientos a partir de ahora depende 
en parte de nosotros, tenemos poco margen, actuemos. Pero actuemos todos; que la magnitud de los números y las grandes estrategias no nos distraigan de que todos somos importantes.

\section{Referencias Bibliográficas}

1. Oteo Iglesias J. La resistencia a Antibióticos: La amenaza de las superbacterias. Editorial Catarata, 2016. ISBN: 978-84-9097-214-4.

2. European Centre for Disease Prevention and Control/European Medicines Agency The bacterial challenge: time to react. 2009. [Consultado el 12 de octubre de 2019] Disponible en: https://www.ecdc.europa.eu/sites/portal/files/media/en/publications/Publications/0909_TER_The_Bacteri al_Challenge_Time_to_React.pdf

3. O'Neil J (cooord.)Antimicrobial Resistance: Tackling a crisis for the health and wealth of nations. 2016. [Consultado el 17 de octubre de 2019] Disponible en: https://amrreview.org/sites/default/files/AMR\%20Review\%20Paper\%20\%20Tackling\%20a\%20crisis\%20for\%20the\%20health\%20and\%20wealth\%20of\%20nations_1.pdf

4. Agencia Española de Medicamentos y Productos Sanitarios (coord.). Plan Nacional frente a la Resistencia a los Antibióticos, PRAN. [Consultado el 17 de septiembre de 2019] Disponible en: http://www.resistenciaantibioticos.es/es

5. Santiago-Rodríguez TM, Fornaciari G, Luciani S, et al. Gut microbiome of an 11th century AD preColumbian Andean mummy. PLoS ONE 2015; 10: e0138135.

6. D'Costa VM, King CE, Kalan L, et al. Antibiotic resistance is ancient. Nature 2011; 477: 457-461.

7. Perry J, Waglechner N, Wright G. The prehistory of antibiotic resistance. Cold Spring Harb Perspect Med. 2016; 6. pii: a025197.

8. Bathoorn E, Tsioutis C, da Silva Voorham JM, et al. Emergence of pan-resistance in KPC-2 carbapenemaseproducing Klebsiella pneumoniae in Crete, Greece: a close call. J Antimicrob Chemother. 2016; 71: 12071212.

9. Mathers AJ, Peirano G, Pitout JD. The role of epidemic resistance plasmids and international high-risk clones in the spread of multidrug-resistant Enterobacteriaceae. Clin Microbiol Rev. 2015; 28:565-591.

10. Oliver A, Mulet $X$, López-Causapé $C$, Juan C. The increasing threat of Pseudomonas aeruginosa high-risk clones. Drug Resist Updat. 2015; 21-22: 41-59.

11. Oteo-Iglesias J. Active surveillance of antimicrobial resistance. Enferm Infecc Microbiol Clin. 2019; 37 Suppl 1:26-31.

12. Woodford N, Wareham DW, Guerra B, et al. Carbapenemase-producing Enterobacteriaceae and nonEnterobacteriaceae from animals and the environment: an emerging public health risk of our own making? J Antimicrob Chemother. 2014; 69: 287-291.

13. Mills MC, Lee J. The threat of carbapenem-resistant bacteria in the environment: Evidence of widespread contamination of reservoirs at a global scale. Environ Pollut. 2019; 255: 113143. doi:10.1016/j.envpol.2019.113143.

14. Kristiansson E, Fick J, Janzon A, et al. Pyrosequencing of antibiotic-contaminated river sediments reveals high levels of resistance and gene transfer elements. PLoS One. 2011; 6: e17038. doi: 10.1371/journal.pone.0017038.

15. Oteo J, Mencía A, Bautista V, et al. Colonization with Enterobacteriaceae-producing ESBLs, AmpCs, and OXA-48 in wild avian species, Spain 2015-2016. Microb Drug Resist. 2018; 24: 932-938.

16. Sun J, Zhang H, Liu YH, et al. Towards understanding MCR-like colistin resistance. Trends Microbiol. 2018; 26: 794-808.

17. Tacconelli E, Carrara E, Savoldi A, et al. Discovery, research, and development of new antibiotics: the WHO priority list of antibiotic-resistant bacteria and tuberculosis. Lancet Infect Dis 2018; 18: 318-327.

18. Magiorakos AP, Srinivasan A, Carey RB, et al. Multidrug-resistant, extensively drug-resistant and pandrug-resistant bacteria: an international expert proposal for interim standard definitions for acquired resistance. Clin Microbiol Infect. 2012; 18: 268-281.

19. Oteo J, Miró E, Pérez-Vázquez M, et al. Evolution of carbapenemase-producing Enterobacteriaceae at the global and national level: what should be expected in the future? Enferm Infecc Microbiol Clin. 2014; 32 Suppl. 4:17-23. 
20. Bathoorn E, Tsioutis C, da Silva Voorham JM, et al. Emergence of pan-resistance in KPC-2 carbapenemaseproducing Klebsiella pneumoniae in Crete, Greece: a close call. J Antimicrob Chemother 2016; 71: 1207-12.

21. European Commission. Special Eurobarometer 478: Antimicrobial Resistance. September 2018. [Consultado el 8 de noviembre de 2019] Disponible en: http://www.google.es/url?sa=t\&rct=j\&q=\&esrc=s\&source=web\&cd=2\&ved=2ahUKEwjOkOGJvu7lAhXFxo UKHRuwB40QFjABegQIAxAC\&url=http $\% 3 \mathrm{~A} \% 2 \mathrm{~F} \% 2 \mathrm{Fec}$.europa.eu $\% 2$ Fcommfrontoffice $\% 2$ Fpublicopinio n\%2Findex.cfm\%2FResultDoc\%2Fdownload\%2FDocumentKy\%2F84386\&usg=AOvVaw2XbUoP0unvmZ VmyibQgFjX

22. Rodríguez-Baño J, Paño-Pardo JR, Alvarez-Rocha L, et al. Programs for optimizing the use of antibiotics (PROA) in Spanish hospitals: GEIH-SEIMC, SEFH and SEMPSPH consensus document. Enferm Infecc Microbiol Clin. 2012; 30: 22.e1-22.e23.

23. Cassini A, Högberg LD, Plachouras D, et al. Attributable deaths and disability-adjusted life-years caused by infections with antibiotic-resistant bacteria in the EU and the European Economic Area in 2015: a population-level modelling analysis. Lancet Infect Dis. 2019; 19: 56-66.

(C) 2019 por los autores; Esta obra está sujeta a la licencia de Reconocimiento 4.0

Internacional de Creative Commons. Para ver una copia de esta licencia, visite http://creativecommons.org/licenses/by-nc-nd/4.0/. 